\title{
Modeling Radiation Damage Effects in 3D Pixel Digitization for the ATLAS Detector
}

\author{
Veronica Wallängen, on behalf of the ATLAS Collaboration \\ Stockholm University, Lawrence Berkeley National Laboratory \\ E-mail: veronica.wallangenecern.ch
}

Silicon Pixel detectors are at the core of the current and planned upgrade of the ATLAS detector. As the detector in closest proximity to the interaction point, these detectors will be exposed to a significant amount of radiation over their lifetime: before the High Luminosity phase of the Large Hadron Collider (HL-LHC) the innermost layers will receive a fluence in excess of $10^{15}$ $\mathrm{n}_{\mathrm{eq}} / \mathrm{cm}^{2}$ and the HL-LHC detector upgrades must cope with an order of magnitude higher fluence integrated over their lifetimes. This work presents the details of a new simulation model that includes radiation damage effects to the 3D Pixel sensors for the ATLAS detector.

The 26th International Workshop on Vertex Detectors

10-15 September, 2017

Las Caldas, Asturias, Spain 


\section{Introduction}

The extreme radiation environment inside the ATLAS detector [1] following the upgrade of the Large Hadron Collider (LHC) to reach a factor of 10 higher luminosity as compared to the current design value requires significantly improved radiation hardness of the particle sensors and readout electronics [2]. This becomes increasingly important closer to the particle interaction point where the hit density is the highest, making 3D silicon sensors [3] interesting candidates to be used in the innermost layers of the upgraded ATLAS inner tracking detector. Radiation exposure causes the formation of defects in the silicon which act as trapping points for the charge generated by the passage of particles in the sensors, thereby resulting in signal loss [4]. This note focuses on modeling of such non-ionizing energy losses in 3D sensors.

3D silicon sensors were first proposed twenty years ago as a more radiation tolerant alternative to traditional planar silicon sensors. In the 3D sensor fabrication process, columns are etched into the silicon substrate to form three-dimensional arrays of electrodes of $\mathrm{p}$-n junctions that penetrate through the sensor bulk perpendicular to the detector surface, rather than being implanted on top of it. The inter-electrode distance can then be decoupled from the sensitive device thickness and hence the drift distance for the charge can be much smaller than for planar sensors. This technology makes the charge carrier collection distances, and thereby collection times, close to one order of magnitude lower compared to the planar counterpart, thus lowering the number of carriers that gets trapped in the sensor bulk as an effect of radiation damage. In addition, the operational voltage can be lowered, resulting in lower power dissipation after heavy irradiation.

3D sensors are already in use in the ATLAS Insertable B-Layer (IBL) [5] and have demonstrated good performance. These devices have a pixel size of $50 \times 250 \mu \mathrm{m}^{2}$, where each pixel has two $\mathrm{n}+$ columns shorted together with an inter-electrode distance of $67 \mu \mathrm{m}$ etched into the $230 \mu \mathrm{m}$ thick p-type silicon substrate. The radiation dose tolerance requirement on the $3 \mathrm{D}$ sensors currently in use is $5 \times 10^{15} \mathrm{n}_{\mathrm{eq}} / \mathrm{cm}^{2}$ [5], while the typical expected fluence at the end of the operation of the HL-LHC is $1 \times 10^{16} \mathrm{n}_{\mathrm{eq}} / \mathrm{cm}^{2}$.

\section{3D pixel sensor digitizer model}

The current ATLAS simulation framework does not account for radiation damage affecting the charge collection process in the pixel sensors. Meanwhile, this is an important feature to be added in the digitization step of the simulation flow, where the energy deposited in each pixel is converted into a digitized detector response, to be able to accurately simulate the detector performance and reconstruct particle trajectories. For this reason, a digitizer model for the 3D sensor coupled to the FE-I4 pixel readout integrated circuit [10] has been developed and an overview of this implementation can be seen in Figure 1.

The 3D digitizer models the trapping of free charge carriers occuring as a consequence of radiation damage. The electric field orientation within the 3D sensor is such that the charge carriers drift towards the electrodes in the $x-y$ plane parallel to the pixel (see Figure 1). Thermal diffusion will cause additional spread of the carriers, thereby giving rise to increased charge sharing between pixels as carriers diffuse into neighboring pixels and thus generate a signal in a pixel different from where they were created. This effect is modeled by displacing the charges through random one-step 
jumps in $x$ and $y$ drawn from a Gaussian distribution where the diffusion length standard deviation is given by

$$
\sigma=\sqrt{\frac{2 \mu(E) k_{B} T t}{q}},
$$

and where $q$ denotes the absolute value of the electron charge, $\mu(E)$ the electric field-dependent mobility, $k_{B}$ the Boltzmann constant, $T$ the temperature and $t$ the time the carrier spends drifting, until either getting trapped or reaching the electrode.

Figure 1: Procedure for the treatment of the effect of each particle "hit" in the digitizer.
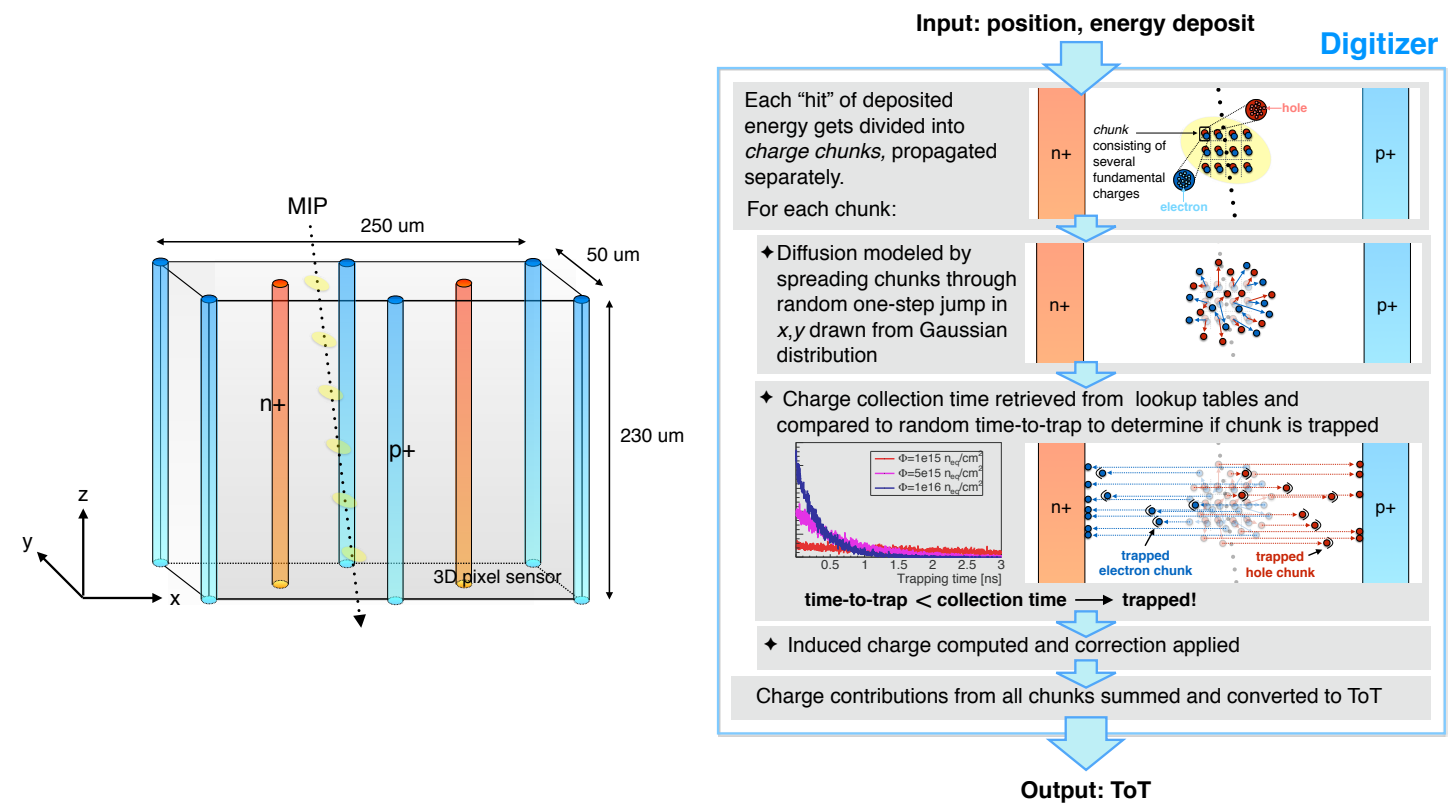

The probability of a charge carrier to make it all the way to the electrode without getting trapped decreases with received fluence $\Phi$ according to

$$
p\left(t_{\text {trap }}\right)=1-e^{-t_{\text {trap }} \kappa \Phi}
$$

where the constant $\kappa=3 \times 10^{-16} \mathrm{~cm}^{2} / \mathrm{ns}$ is experimentally determined [7] and $t_{\text {trap }}$ is the time the carrier spends drifting until getting trapped. In order to determine whether a carrier is trapped, this time is randomly selected from an exponential distribution with mean value $1 / \kappa \Phi$ and compared to the expected time it would take the carrier to reach the electrode

$$
t_{\mathrm{el}}=\int_{P_{0}}^{n+, p+} \frac{1}{\mu(E)^{e, h} E} \mathrm{~d} s,
$$

estimated based on the initial position where the carrier was created $P_{0}=\left(x_{0}, y_{0}\right)$, by integrating the equation of motion over the drift path towards the electrode using the drift velocity $v_{\text {drift }}=$ $\pm \mu^{e, h}(E) E$ determined from the corresponding carrier-dependent mobility $\mu^{e, h}$, which is parameterized by the electric field $E$ and temperature [8].

As the carriers drift along the electric field lines and simultaneously diffuse, their movement will induce a current on neighboring electrodes and will thus give rise to a signal regardless of 
whether they physically reach the electrode or not. The amount of charge induced can be estimated using a geometry-dependent weighting potential referred to as the Ramo potential [9]. The charge induced by a carrier at position $(x, y)$ after having drifted along a path starting at initial location $\left(x_{0}, y_{0}\right)$ will be the difference in Ramo potential $\Delta \phi$ between these two positions multiplied by the elementary carrier charge $q$, so that $Q=-q \Delta \phi=-q\left[\phi(x, y)-\phi\left(x_{0}, y_{0}\right)\right]$.

\section{TCAD simulations}

Detailed data about the Ramo potential and the electric field profile within the sensor at different levels of irradiation is gathered from Technology Computer-Aided Design (TCAD) simulations using the Perugia model [10]. Fluences considered in this study along with corresponding bias voltages to model full depletion are listed in Table 1. From the electric field data, the expected times to reach the electrode for electrons and holes respectively can easily be pre-calculated according to equation (2.3) prior to runtime and stored in histograms that function as lookup tables to quickly be retrieved for any initial position within the pixel, which results in a faster digitizer performance (see Figure 2 and Figure 3).

Table 1: Simulated fluences and corresponding bias voltages.

\begin{tabular}{|c|c|c|c|c|c|c|c|}
\hline Fluence $\left[\mathbf{n}_{\mathrm{eq}} / \mathbf{c m}^{2}\right]$ & $1 \times 10^{14}$ & $2 \times 10^{14}$ & $5 \times 10^{14}$ & $1 \times 10^{15}$ & $5 \times 10^{15}$ & $6 \times 10^{15}$ & $1 \times 10^{16}$ \\
\hline Bias voltage [V] & -20 & -30 & -40 & -50 & -160 & -190 & -260 \\
\hline
\end{tabular}
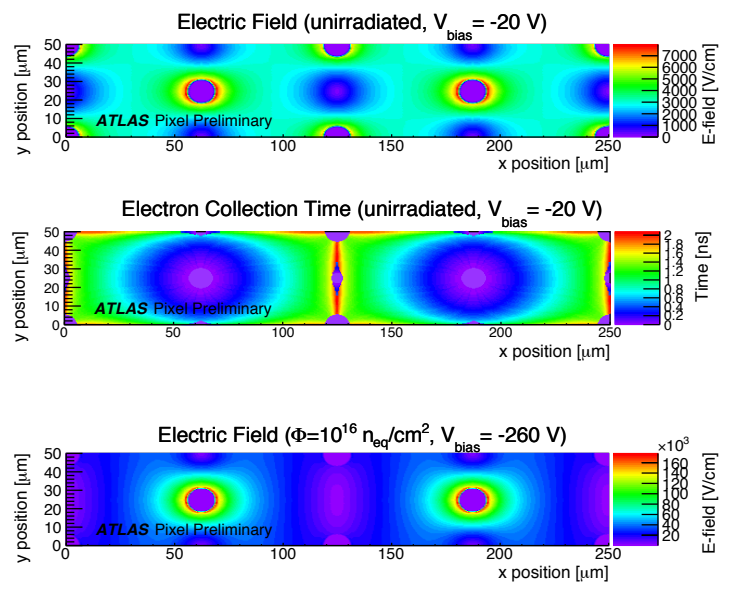

Electron Collection Time $\left(\Phi=10^{16} n_{\text {eq }} / \mathrm{cm}^{2}, \mathrm{~V}_{\text {bias }}=-260 \mathrm{~V}\right)$

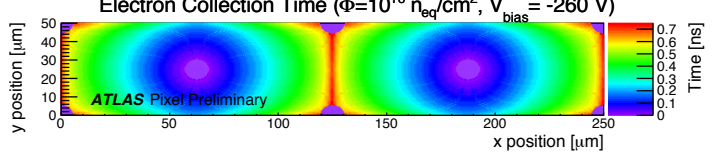

Figure 2: Electric field in unirradiated pixel sensor and sensor irradiated to $10^{16}$ $\mathrm{n}_{\mathrm{eq}} / \mathrm{cm}^{2}$ along with corresponding electron collection time maps.
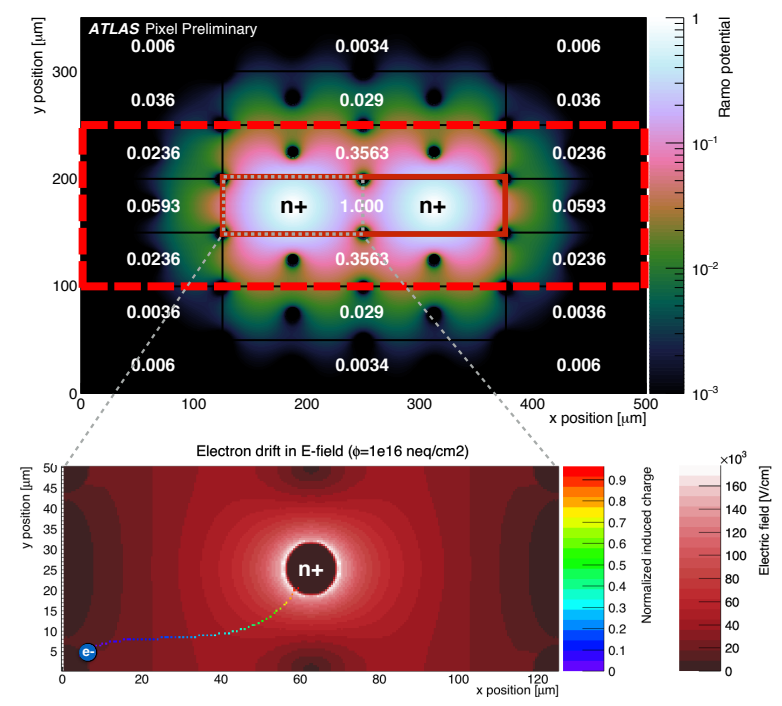

Figure 3: Top: Ramo potential in primary pixel and neighbors. Maximum fraction of induced charge on electrodes in primary pixel due to carrier moving within neighboring pixel displayed in bold. Bottom: Average fraction of induced charge from electron at different trapping locations. 


\section{Model correction}

The induced charge $Q$ will be proportional to the energy loss of a particle as it passes through silicon, which causes $\sim 80$ electron-hole pairs per micron to be produced as a result. For efficient digitization, each fundamental charge is not treated separately, but instead the energy deposited in each hit is divided into "chunks" of several fundamental charges that are collectively propagated through the digitizer. This simplification will not impact the average amount of induced charge $\langle Q\rangle$ but will cause enlarged fluctuations, something which is compensated for by applying a correction to account for the fact that the charge chunks are not fundamental according to

$$
Q \rightarrow Q+\frac{1}{\sqrt{n}}(Q-\langle Q\rangle)
$$

The correction factor scales with the number of electron-hole pairs in the chunk $n(n \sim 10)$ and the average charge induced by the collection of particles starting out at position $\left(x_{0}, y_{0}\right)$ can be obtained by integrating the equation of motion as the electrons and holes drift along the electric field lines towards the $\mathrm{n}+$ electrode and $\mathrm{p}+$ electrodes respectively such that

$$
\langle Q\rangle=q n\left(\int_{P_{0}}^{n+} p^{(e)}\left[\left(x_{0}, y_{0}\right) \rightarrow(x, y)\right]\left[\phi(x, y)-\phi\left(x_{0}, y_{0}\right)\right] \mathrm{d} s-\int_{P_{0}}^{p+} p^{(h)}\left[\left(x_{0}, y_{0}\right) \rightarrow(x, y)\right]\left[\phi(x, y)-\phi\left(x_{0}, y_{0}\right)\right] \mathrm{d} s\right),
$$

where $p^{(e),(h)}\left[\left(x_{0}, y_{0}\right) \rightarrow(x, y)\right]$ is the probability of the carrier to reach point $(x, y)$ without getting trapped. The digitization method uses lookup tables to determine the final position of the carrier as well as the Ramo potential at the initial and final position to compute the amount of charge induced in the closest and neighboring electrodes as illustrated in Figure 4.

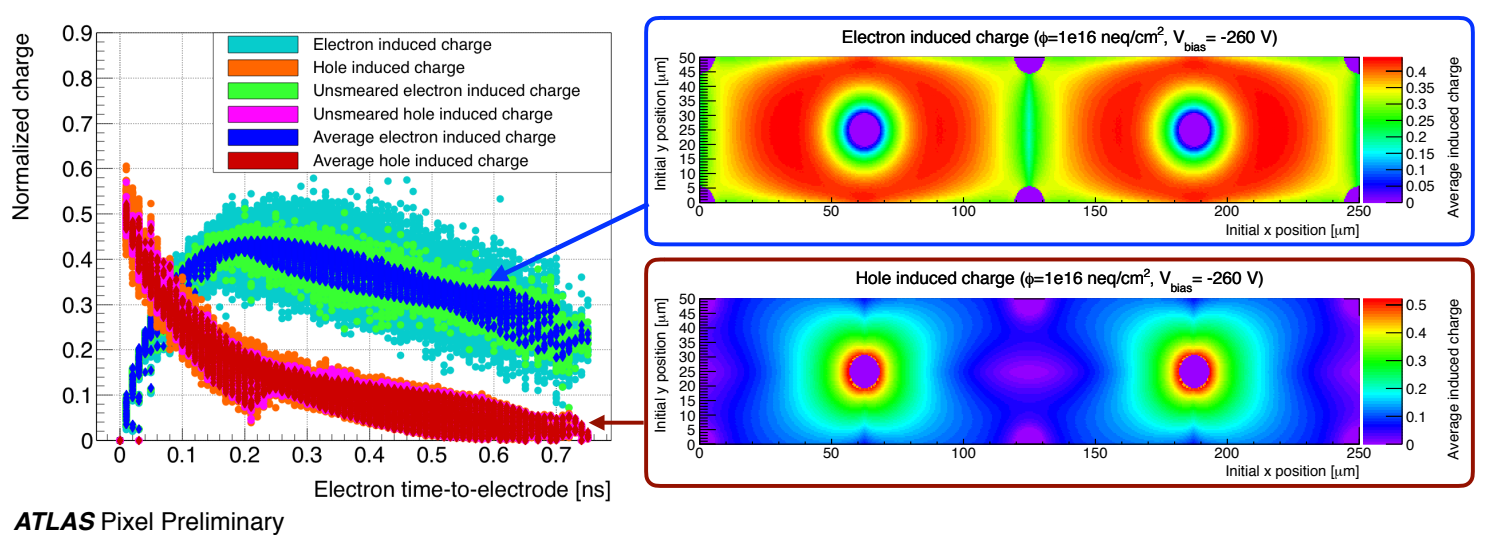

Figure 4: Charge collected normalized to total charge deposited as function of the electron collection time and displayed with and without correction ("unsmearing"), along with corresponding histograms to retrieve the average fraction of induced charge for electrons and holes respectively.

\section{Results and conclusion}

The ratio of the charge collected after irradiation to the charge collected before irradiation is referred to as the charge collection efficiency, a parameter that provides understanding of the signal 
loss and performance degradation during the detector lifespan. The charge collection efficiency as function of fluence in the 3D digitizer model is displayed in Figure 5. The data points have been extracted by performing a Landau fit convoluted with a Gaussian on the ToT distributions and correspond to the most probable value. An additional fit to these data points indicates an exponential decrease in charge collection efficiency with fluence. These results agree with previous experimental studies reported in [11].

Figure 5: Charge collection efficiency for different fluences produced using the 3D pixel digitizer model. Charge collection efficiency

\section{References}

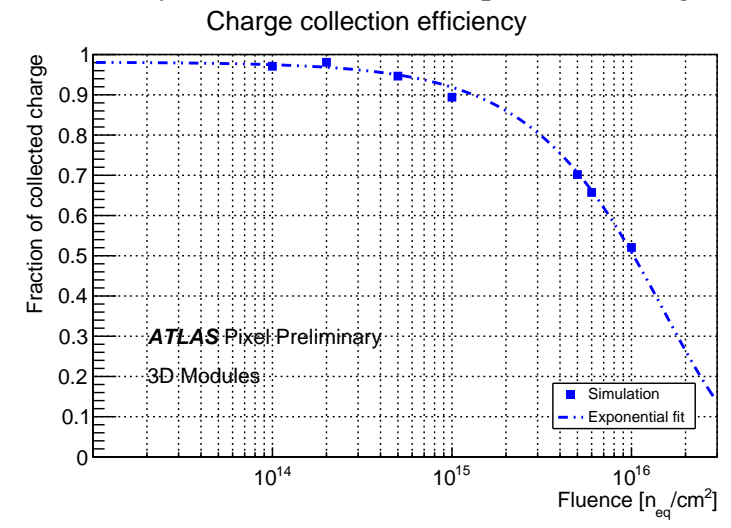

[1] ATLAS Collaboration, The ATLAS Experiment at the CERN Large Hadron Collider, JINST 3 S08003 (2008).

[2] ATLAS Collaboration, Technical Design Report for the ATLAS Inner Tracker Strip Detector (2017) [CERN-LHCC-2017-005].

[3] S.I. Parker, C.J. Kenney. and J. Segal, 3D - A proposed new architecture for solid-state radiation detectors, Nucl. Instr. and Meth. 395 328-343 (1997).

[4] M. Moll, Radiation damage in silicon particle detectors: Microscopic defects and macroscopic properties, PhD thesis: Hamburg U. (1999).

[5] ATLAS Collaboration, ATLAS Insertable B-Layer Technical Design Report (2010) [CERN-LHCC-2010-013].

[6] M. Garcia-Sciveres et al., The FE-I4 Pixel Readout Integrated Cirtcuit in proceedings of 7th International 'Hiroshima' Symposium on the Development and Application of Semiconductor Tracking Detectors (HSTD-7), Nucl. Instr. and Meth. A636 S155-S159 (2011).

[7] G. Alimonti et al., A study of charge trapping in irradiated silicon with test beam data, ATL-INDET-2003-014 (2003) [cds.cern.ch:685542].

[8] C. Jacoboni et al., A review of some charge transport properties of silicon, Solid-State Electronics 20 77 (1977).

[9] W. Shockley, Currents to Conductors Induced by a Moving Point Charge, Journal of Applied Physics 9635 (1938).

[10] F. Moscatelli et al., Combined Bulk and Surface Radiation Damage Effects at Very High Fluences in Silicon Detectors: Measurements and TCAD Simulations, IEEE Transactions on Nuclear Science $\mathbf{6 3}$ 2716 (2016).

[11] C. Da Via et al., 3D active edge silicon sensors: device processing, yield and QA for the ATLAS IBL production, Nucl. Instrum. Meth A 699 (2013) 18. 